\title{
The effects on student health of interventions modifying the school environment: systematic review
}

\author{
C Bonell, ${ }^{1} \mathrm{H}$ Wells, ${ }^{2}$ A Harden, ${ }^{3} \mathrm{~F}$ Jamal, ${ }^{3}$ A Fletcher, ${ }^{4} \mathrm{~J}$ Thomas $^{5}{ }^{5}$ R Campbell, ${ }^{6}$ \\ M Petticrew, ${ }^{2}$ M Whitehead, ${ }^{7}$ S Murphy, ${ }^{4}$ L Moore $^{4}$
}

- Additional material is

published online only. To view please visit the journal online (http://dx.doi.org/10.1136/jech2012-202247).

${ }^{1}$ Department of Social Policy and Intervention, Centre for Evidence Based Intervention, University of Oxford, Oxford, UK

${ }^{2}$ Department of Social and Environmental Health Research, London School of Hygiene and Tropical Medicine, London, UK ${ }^{3}$ Institute for Health and Human Development, University of East London, London, UK

${ }^{4}$ DECIPHer UKCRC Public Health Research Centre of Excellence, School of Social Sciences, Cardiff University, Cardiff, UK

${ }^{5}$ Department of Childhood, Families and Health, Institute of Education, University of London, London, UK ${ }^{6}$ School of Social and Community Medicine, University of Bristol, Bristol, UK 7 Institute of Psychology, Health and Society, University of Liverpool, Liverpool, UK

\section{Correspondence to} Professor Chris Bonell, Department of Social Policy and Intervention, Centre for Evidence Based Intervention, University of Oxford, 32 Wellington Square, Oxford OX1 2ER, UK;

christopher.bonell@spi.ox.ac.uk

Received 5 December 2012 Revised 16 April 2013 Accepted 17 April 2013 Published Online First 16 May 2013

To cite: Bonell $\mathrm{C}$, Wells $\mathrm{H}$ Harden $\mathrm{A}$, et al. J Epidemiol Community Health 2013;67:677-681.

\section{ABSTRACT}

Background Owing to the limited effectiveness of traditional health education curricula in schools, there is increasing interest in interventions aiming to promote young people's health by modifying the school environment. Existing systematic reviews cannot determine whether environmental intervention is effective because they examine interventions combining environmental modifications and traditional health education. This gap is significant because schoolenvironment interventions are complex to implement and may be sidelined in underfunded and attainment-focused school systems without evidence to support such an approach. This systematic review examined the effectiveness of school-environment interventions without health-education components on student health and inequalities.

Methods This was a systematic review of experimental/ quasi-experimental studies of school-environment interventions. Sixteen databases were searched, eliciting 62329 references which were screened, with included studies quality assessed, data extracted and narratively synthesised.

Results Sixteen reports of 10 studies were included, all from the USA and the UK. Five evaluations of interventions aiming to develop a stronger sense of community and/or improve relationships between staff and students suggested potential benefits particularly regarding violence and aggression. Two trials of interventions enabling students to advocate for changes in school catering and physical activity reported benefits for physical activity but not diet. Three evaluations of improvements to school playgrounds offered weak evidence of effects on physical activity.

Conclusions School environment interventions show the potential to improve young people's health particularly regarding violence, aggression and physical activity. Further trials are required to provide a stronger and more generalisable evidence base.

\section{INTRODUCTION}

Health risk-behaviours increase during adolescence and are subject to marked variations by social status. ${ }^{1}$ There is increasing academic ${ }^{1}{ }^{2}$ and policy interest ${ }^{3}$ in interventions aiming to promote young people's health by ensuring that the wider school environment (SE), in terms of both the physical and social environment, supports healthy behaviours. This reflects awareness that traditional health-education curricula struggle to find a place in school timetables ${ }^{4}$ and show disappointing results $^{5-9}$ and interest in socioecological determinants of health, of which SE is one important element. ${ }^{10}$ If effective, such interventions might represent a pragmatic and efficient means of addressing multiple intercorrelated risk behaviours. ${ }^{11} 12 \mathrm{SE}$ interventions can address health behaviours directly, for example, via smoking policies, and indirectly via addressing 'upstream' risk factors, such as school disengagement. ${ }^{13}$

In North America, 'Coordinated School Health Programs' (CSHP) include SE components alongside health-education curricula, while such approaches have been termed 'Health-Promoting Schools' interventions (HPS) in Europe and elsewhere. ${ }^{4}$ We currently do not know whether modifying the SE is an 'active ingredient' in such programmes. Existing reviews are unable to answer this question because although systematic reviews of HPS/CSHP report positive findings, ${ }^{4}{ }^{14} 15$ they cannot distinguish the effects of SE and health-education curricula. And while systematic reviews of multilevel studies conclude that various school-level factors are associated with better health outcomes, ${ }^{16}{ }^{17}$ these are vulnerable to residual confounding and may focus on school factors not readily open to intervention.

Assessing whether modifying the SE is an 'active ingredient' in CSHP/HPS interventions is important because SE intervention requires substantial staff time. Without evidence of effectiveness, SE interventions may become marginalised in underfunded and attainment-focused school systems. In the UK, the coalition government no longer directs schools to engage in HPS programmes nor inspects schools on how they promote health. ${ }^{18}$ Examining whether SE interventions are effective through randomised controlled trials (RCTs) is also scientifically important as these designs enable rigorous assessment of causation in testing socioecological theories of health as applied to the school setting. ${ }^{10}$ The theory of human functioning and school organisation ${ }^{10}$ suggests that schools can promote health through their 'core business' of teaching, pastoral care and discipline by increasing student commitment to learning and the school community, thereby reducing investment in antischool peer groups and risk behaviours. This theory suggests that schools can achieve this by improving staff-student relationships, and by ensuring that teaching and decision-making are more student-centered. Our systematic review therefore sought to examine the effectiveness of SE interventions addressing such factors on student health and health inequalities. This question is elaborated below.

\section{METHODS}

Our review followed a protocol, available online. ${ }^{19}$ Our review of SE interventions was part of a larger 
project mapping and synthesising evidence on how the SE influences health. In stage 1 , we descriptively mapped the relevant literature. Sixteen bibliographic databases were searched between 30 July and 23 September 2010, with no limits on language or date. The published protocol and the full project report $^{20}$ provide a complete list of databases searched (see online supplementary appendix). Our search used naturallanguage phrases to maximise sensitivity. Our first searches involved terms for setting (school), population (children), intervention (SE) and health outcomes, linked by 'and'. Additional searches used various phrases related to HPS. These retrieved 82775 references, which were uploaded into EPPI-Reviewer 4 software $^{21}$ and duplicates removed, leaving 62329 references. We used stage- 1 exclusion criteria (detailed in our protocol) to screen references on title/abstract. These were piloted and then applied by six reviewers, with a random sample of $10 \%$ being double-screened to check for consistency (>1\% disagreement). Our map included 1144 references, ${ }^{20}$ which was discussed with academic, policy and youth stakeholders to inform priorities for stage 2 .

Stage 2 involved several in-depth reviews, including that reported here. Informed by stakeholder views, health outcomes encompassed violence, sexual health, substance use, obesity, physical activity and diet, mental health, sun protection and injury. Interventions addressed SE in terms of what our stakeholders termed schools' 'core business': school organisation and management; teaching, discipline and pastoral care and the physical environment. We did not focus on teachers' health or on interventions involving only catering or physical exercise lessons since these are the subject of ongoing and existing reviews, respectively. ${ }^{22} 23$ We also excluded studies of interventions combining SE and health-education components.

The 524 references in our map which had been coded as experimental (cluster RCTs) or quasi-experimental (nonrandomised cluster-level studies with concurrent comparison groups) studies of SE interventions were rescreened using additional exclusion criteria (figure 1) reflecting the stakeholder priorities listed above. Two reviewers independently double-screened these references based on the title/abstract where possible and full reports where the titles/abstracts did not provide sufficient information. We extracted data from included studies on: intervention; resources; comparison group; site and population; sampling; schools and individuals per arm; data collection; analysis and results. All included reports were quality-assessed using adapted EPPI-Centre tools ${ }^{24}$ to weight interpretation in our narrative synthesis. Data-extraction and quality-assessment tools were piloted before being applied by one reviewer and checked by another, with any differences being settled by discussion. Studies were judged to be well conducted overall if they randomly allocated schools, and had no more than $30 \%$ attrition with $10 \%$ difference between arms. We determined that statistical meta-analysis of intervention effects necessitated three or more studies homogeneous regarding intervention and methods ${ }^{25}$ and did not find this. Therefore, the findings were narratively synthesised.

\section{RESULTS}

\section{Overview of included studies}

Stage- 2 screening excluded all but $10^{26-35}$ of the 524 potentially relevant studies identified from stage 1 (figure 1 ). Three other included studies were sourced from experts ${ }^{36-38}$ and three via checking reference lists of included reports. ${ }^{39-41}$ These 16 study reports arose from 10 separate studies published between 1988 and 2010 and conducted in elementary, middle and secondary schools in the USA and the UK (see online supplementary table S1). Study quality is summarised in online supplementary table S2. Six were RCTs, ${ }^{26-32} 363739$ although this was somewhat compromised in one study ${ }^{26}$ by the replacement of a dropout school with a non-randomised substitute. Four studies were quasi-experimental. $^{33-35} \quad 38 \quad 4041$ Interventions fell into three categories: enabling staff and students to develop a stronger sense of community and/or better relationships to reduce aggression and other risk behaviours; enabling students to advocate for changes to school catering and physical-activity routines (included because of their student-empowerment element); and improving school playgrounds.

\section{Interventions enabling staff and students to develop a stronger sense of community and/or better relationships}

Nine reports of five evaluations (all but one an RCT) examined interventions enabling staff and students to build stronger school communities and relationships. These were conducted in a range of school settings. All except the Healthy School Ethos (HSE) intervention (UK) were conducted in the USA. Taken together, these studies suggest the potential of such interventions to bring about benefits particularly regarding violence and aggression.

The Child Development Project (CDP) $\begin{array}{llll}30 & 36 & 37 & 39 \\ \text { modified }\end{array}$ classroom-management and teaching. It was reported as having positive effects on three of four measures of cognitive-social problem-solving in conflict situations, as well as teacherreported student solving of minor interpersonal problems. No effects were reported on self-esteem. It also had benefits for loneliness/social dissatisfaction and social anxiety. Results should be interpreted with caution because: baseline data were not collected; no account was made for data clustering; and attrition was high and differed between arms.

The Aban Aya Youth Project (AAYP) and HSE projects modified management and pastoral care in elementary/middle schools in Chicago. ${ }^{29}$ The RCT comprised three arms: school/ community intervention (SCI), including SE/health education curriculum components; social development curriculum (SDC)only, a health education curriculum; and a no-intervention comparison group. Comparing SCI with SDC arms enabled us to distinguish the effects of the SE component, hence its inclusion. Boys in schools receiving SCI versus those receiving SDC-only experienced non-significant benefits regarding violence, recent sexual intercourse and condom use. Only for two non-health outcomes were differences significant. Among girls, there was a significant benefit for self-reported condom use but no other effects. This was a well-conducted RCT with little risk of bias. HSE delivered in secondary schools ${ }^{38}$ was associated with significantly higher rates of feeling safe at school but no significant differences in self-reported teasing of others, hurting of others, being in a fight, being teased, threatened or hurt in school or attitudes regarding substance use. This was a small quasi-experimental study, so results should be treated with caution.

The Creating a Peaceful School Learning Environment (CAPSLE) and PeaceBuilders (PB) interventions modified discipline. CAPSLE was delivered in elementary schools. ${ }^{31}$ The RCT involved three arms, comparing CAPSLE to a psychiatric intervention and a no-intervention comparison. We review differences between the CAPSLE and no-treatment arms. Benefits were reported regarding: peer-reported victimisation and aggression; self-reported aggression and empathy and peerreported aggressive by-standing and 'helpful by-standing' (intervening to prevent aggression). However, for peer-reported 
Figure 1 Flow of literature outcome evaluation synthesis (stage 2: in-depth synthesis).

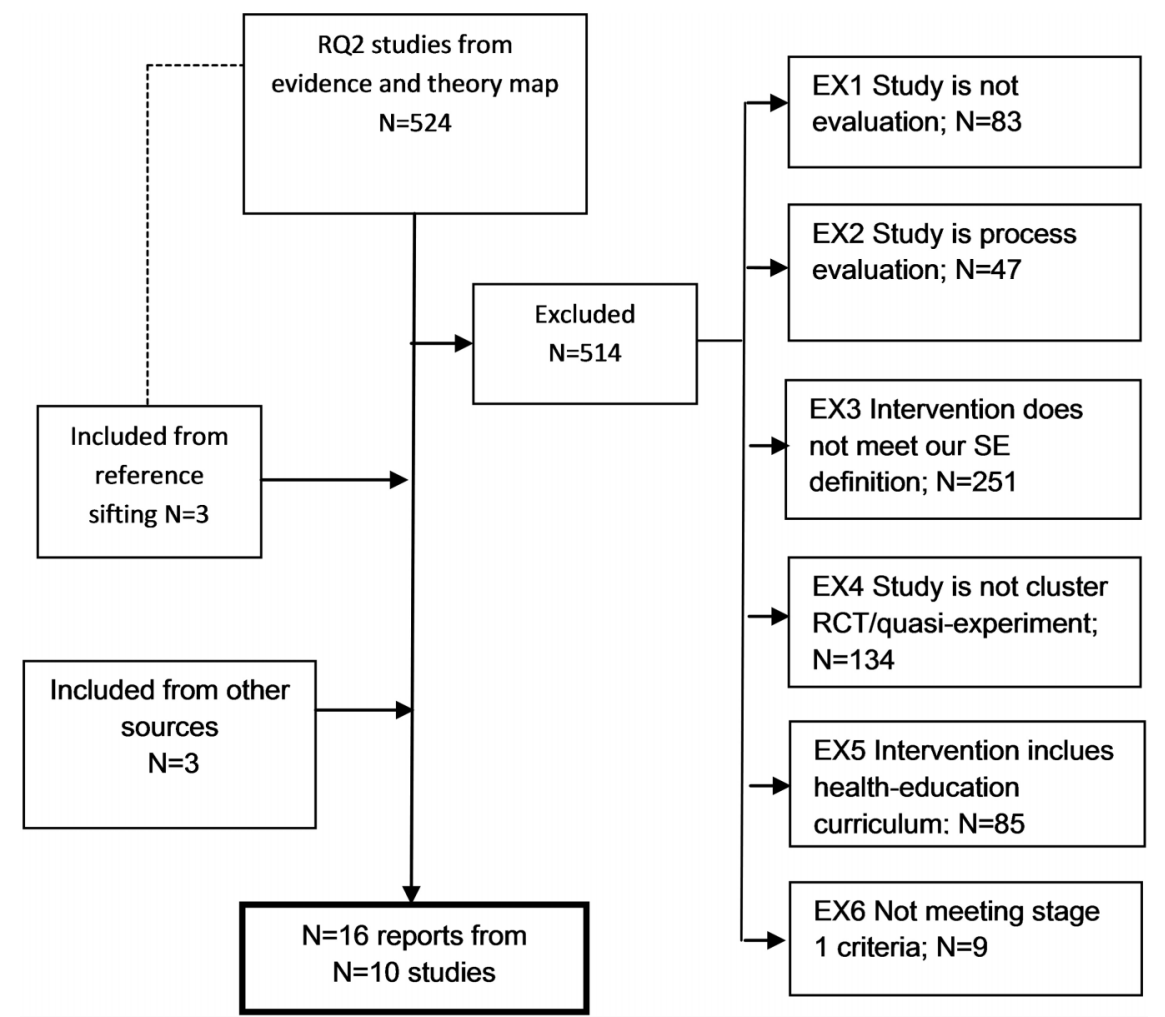

helpful by-standing, these effects were not sustained. There were no effects for self-reported victimisation or self-reported beliefs in the legitimacy of aggression. This RCT had high attrition which differed between groups and no account was taken of data clustering. Evaluated in a well-conducted study, PB was delivered in elementary schools. ${ }^{26} 32$ The intervention had significant effects on teacher-rated aggression among some grades but there were no intervention effects on child self-reported aggression. Effects for teacher-rated aggression were larger for students with higher baseline aggression, although interaction was not tested. Also, there were intervention effects on injury/ fighting-related visits to the school nurse.

\section{Interventions enabling students to advocate for changes to school catering and physical activity}

Two US experimental studies assessed interventions which combined changes to middle schools' food and physical-activity environments alongside actions to empower students to contribute to these changes. These were well-conducted studies, which reported intervention benefits for some measures of student physical activity but no effects on diet. Although it is not possible to conclude definitively whether student empowerment was an 'active ingredient', mediation analysis in one study ${ }^{28}$ suggested that it may have been important.

Healthy Youth Places was conducted in middle schools. ${ }^{28}$ The intervention was associated with increases in some but not all measures of physical activity. There were significant increases in student efficacy to ensure that schools were creating supportive environments for physical activity as well as their efficacy to be physically active, but not in their efficacy with regard to parents/ peers creating supportive environments for physical activity. The intervention did not have effects on healthy eating other than students' self-efficacy to eat 5-7 portions of fruit and vegetables, and group norms supportive of fruit-and-vegetable consumption. Effects did not vary by gender, race or socioeconomic status. A measure of student proxy efficacy appeared to mediate intervention effects on physical activity, suggesting its causal importance. This trial was well conducted. The Middle-School Physical Activity and Nutrition intervention was delivered in middle schools. ${ }^{27}$ There was a significant intervention effect on the observed physical activity overall for boys but not girls, although the interaction tests were not undertaken. There were no significant differences in consumption of fat, self-reported physical activity or sedentary behaviours or parental fat avoidance. There was a significant reduction in body mass index among boys but not girls. This was a well-conducted trial.

\section{Intervention aiming to improve school playgrounds}

Three quasi-experimental evaluations (five reports) examined the effects of interventions to improve playgrounds in English elementary schools. Overall, given the limitations of these three studies, we must conclude that there is not currently strong evidence for these interventions, despite a consistent suggestion that they have potential, and may be more effective for younger children and when the recess is longer. One intervention improved playgrounds and provided sporting equipment in elementary schools. ${ }^{33-35}$ There were unexplained inconsistencies between study reports in how many schools participated at each follow-up. The study suggests few initial effects but more consistent effects across various measures at 6 months, with less consistency at 12 months. There was also evidence of greater effects for younger children and longer recess periods. Findings from this study should be interpreted cautiously because of its non-random design and unclear reporting of follow-up data. Two studies report on interventions to improve playground surface markings in primary schools in north-west England. One study examined an intervention in which the surface markings were designed by students, involving one intervention and one comparison school. ${ }^{40}$ The other involved an outside designer, comparing four intervention and four control schools. ${ }^{41}$ The 
first study suggested benefits for physical activity, which may be greater for younger children and where the recess was longer. The second study suggested significant effects immediately after intervention on physical activity. Findings from both these studies should be interpreted cautiously because of their nonrandom designs, variable rates of attrition between arms and failure to account analytically for cluster.

\section{DISCUSSION}

\section{Summary of key findings}

Six experimental studies (10 reports) and four quasi-experimental studies (six reports) were included. These addressed most aspects of schools' 'core business': CDP changed classroom management and teaching; the AAYP and HSE projects modified management and pastoral care; CAPSLE and PB modified discipline and the playground interventions modified physical environment. Reports examined a broad variety of outcomes but with more attention to violence, healthy eating and physical activity than, for example, substance use and sexual health. Studies provided few data relevant to health inequalities.

The studies of interventions enabling staff and students to develop a stronger sense of community and/or better relationships suggested potential benefits particularly regarding violence and aggression. However, this evidence is not definitive given that most studies were subject to methodological limitations and that the strongest evaluation in this category of the AAYP intervention reported the least promising results when comparing the SE-plus-health-education-curriculum arm with the curriculum-only arm. However, although the SE component was the focus of this review, the AAYP investigators reported that it may have acted synergistically with the health-education curriculum because there were substantially larger effect sizes of the combined intervention than the curriculum-only intervention when each was compared with the no-treatment control. $^{29}$

The two well-conducted RCTs of interventions enabling students to advocate for changes to school catering and physical-activity environments reported benefits for some measures of student physical activity but not healthy eating. Although the evidence for the effectiveness of these interventions is somewhat stronger, it is not possible to conclude definitively whether student empowerment was an active ingredient. Finally, given the methodological limitations of the quasiexperimental studies of interventions to improve school playgrounds, we must conclude that there is not currently strong evidence for the effects of these interventions, despite a consistent suggestion that these interventions have potential and may be more effective for younger children and when the recess is longer.

Regarding effects on health inequalities, seven reports examined subgroup effects but only one examined effects by SES. Subgroup reporting was done inconsistently and rarely involved tests for interaction, so these studies provide little information on the likely impacts on health inequalities. The AAYP intervention was delivered in deprived areas; suggesting that SE interventions addressing low-income communities are feasible.

\section{Deviations from protocol and other limitations}

There were three minor deviations from the protocol. ${ }^{19}$ First, owing to the lack of time, we did not undertake forward citation checking. Second, owing to recruitment delays, we did not, in our initial sifting of references, have three reviewers working in parallel comparing screening results in batches.
Instead, sifting was done by the principal investigator working in parallel with five researchers separately. Third, we did not extract reports of authors' conclusions because these would not inform our synthesis. Regarding other limitations, we did not prespecify which outcomes were primary, but, to avoid inappropriate interpretation of significant findings, our narrative synthesis gave equal weight to findings of no significant association. This was intentionally an exploratory review which examined a heterogeneous group of interventions, populations and outcomes; narrowing down on a more homogeneous body of evidence would have run counter to our aim, informed by stakeholder consultation, to examine whether various modifications to SE might have a range of benefits for student health. We developed detailed guidance to the research team to ensure consistency regarding which outcomes and interventions met our inclusion criteria. While our decisions might be challenged (eg, conflict resolution was included, whereas students' ability to share play equipment was not included; interventions addressing classroom management across a school were included but curricula to improve behaviour within particular classrooms were not), our criteria and guidance did nonetheless ensure consistency. Finally, like all systematic reviews, ours is vulnerable to publication bias.

\section{Implications for research and policy}

This review suggests that interventions to modify SE in terms of organisation and management, teaching, discipline and pastoral care and physical environment have the potential to improve young people's health in a range of domains. Our findings offer some tentative evidence in support of the theory of human functioning and school organisation ${ }^{10}$ in that interventions which aimed by various means to improve relationships between staff and students and to ensure that students were more engaged in the classroom and involved in decision-making across the school appeared to provide some benefits particularly regarding reduced violence and aggression and increased physical activity. Our review provides general evidence of the plausibility of effects rather than enabling the probability of effect sizes to be rigorously estimated through meta-analysis.

More rigorous evidence is required to determine the effects of SE interventions. We recommend that more RCTs examine the effects of SE components with and without additional health-education curricula to assess active ingredients and possible synergies. Our review has identified particular gaps, such as studies outside the USA, and interventions focused on outcomes other than violence, physical activity and healthy eating. We would suggest that evaluations of SE intervention should also examine educational outcomes since they may be critical in the broader adoption of such interventions. Finally, future trials should also examine subgroup effects to assess potential impacts on health inequalities, include integral process evaluations to examine fidelity and acceptability, and assess cost-effectiveness. Four of the included studies included process evaluations, ${ }^{20}$ but none included economic evaluations.

We should stress that our focus has been on the effects of modifying 'core', but nonetheless particular, aspects of SE; our results should not be viewed as an indication of the overall evidence base for HPS or CSHP interventions, which include SE alongside health-education curricula (for which a Cochrane review is ongoing), or of interventions aiming to modify SE in other ways such as addressing catering or active transport, which are reviewed elsewhere. ${ }^{22} 23$ 


\section{What is already known on this subject?}

- There is increasing interest in the health benefits of modifying the school environment (SE).

- But existing systematic reviews cannot address or isolate the effects of this because they examine interventions which combine environmental change and traditional health education.

\section{What this study adds?}

- Interventions modifying SE in terms of organisation, teaching, pastoral care and discipline, or physical environment showing the potential to improve young people's health particularly regarding violence, aggression and physical activity.

Acknowledgements We would like to thank Val Hamilton, Carol Vigurs, Dr Rebecca Langford, Jeff Brunton, Sergio Graziosi, Dr Alison O'Mara-Eves, Zahida Suleman and Juan Daniel Kennedy for their contribution to the project.

Contributors $C B$ conceived the study and directed the project. He was responsible for the design and methods, sifting and data extraction, synthesis and write-up. HW was involved in the sifting, data extraction and write-up. AH co-directed the project and was involved in the sifting and data extraction. FJ and AF were involved in the sifting and write-up. RC, MP, MW, SM and LM advised on the methods and comments on drafts.

Funding This project was funded by the National Institute for Health Research Public Health Research (NIHR PHR) Programme (project number 09/3002/08). Visit the PHR Programme website for more information. The views and opinions expressed therein are those of the authors and do not necessarily reflect those of the PHR Programme, NIHR, NHS or the Department of Health.

\section{Competing interests None.}

Provenance and peer review Not commissioned; externally peer reviewed.

\section{REFERENCES}

1 Viner RM, Ozer EM, Denny S, et al. Adolescence and the social determinants of health. Lancet 2012:379:1641-52.

2 Kidger J, Araya R, Donovan J, et al. The effect of the school environment on the emotional health of adolescents: a systematic review. Pediatrics 2012;129:925-49.

3 Lister-Sharp DJ, Chapman S, Stewart-Brown S, et al. Health promoting schools and health promotion in schools: two systematic reviews. Health Technol Assess 1999;3.

4 Fletcher A, Bonell C, Sorhaindo A. "We don't have no drugs education": the myth of universal drugs education in English secondary schools? Int I Drug Policy 2010:21:452-8

5 DiCenso A, Guyatt G, Willan A, et al. Interventions to reduce unintended pregnancies among adolescents: systematic review of randomised controlled trials. Br Med J 2002;324:1426-34.

6 Faggiano $F$, Vigna-Taglianti FD, Versino $E$, et al. School-based prevention for illicit drugs use. Cochrane Database Syst Rev 2005;(2): CD003020.

7 Foxcroft DR, Ireland D, Lowe G, et al. Primary prevention for alcohol misuse in young people. Cochrane Database Syst Rev 2002;(2):CD003020.

8 Thomas R, Perera R. School-based programmes for preventing smoking (Cochrane Review). Cochrane Database Syst Rev 2006;3:CD001293.

9 Wells J, Barlow J, Stewart-Brown S. A systematic review of universal approaches to mental health promotion in schools. Health Educ 2003;103:197-220.

10 Markham WA, Aveyard P. A new theory of health promoting schools based on human functioning, school organisation and pedagogic practice. Soc Sci Med 2003;56:1209-20.

11 Jackson C, Sweeting $\mathrm{H}$, Haw $\mathrm{S}$. Clustering of substance use and sexual risk behaviour in adolescence: analysis of two cohort studies. Br Med J Open 2012;2:e000661.

12 Resnick MD, Bearman PS, Blum RW, et al. Protecting adolescents from harm: findings from the national longitudinal study on adolescent harm. J Am Med Assoc 1997;278:823-32.

13 Perra O, Fletcher A, Bonell C, et al. School-related predictors of smoking, drinking and drug use: evidence from the Belfast Youth Development Study. J Adolesc 2012;35:315-24.
14 Fletcher A, Bonell C, Hargreaves J. School effects on young people's drug use: a systematic review of intervention and observational studies. J Adolesc Health 2008:42:209-20.

15 Langford R, Campbell R, Magnus D, et al. The WHO Health Promoting School framework for improving the health and well-being of students and staff. Cochrane Database Syst Rev 2011(1):CD008958.

16 Aveyard A, Markham WA, Cheng KK. A methodological and substantive review of the evidence that schools cause pupils to smoke. Soc Sci Med 2004;58:2252-65.

17 Evans-Whipp T, Beyers JM, Lloyd S, et al. A review of school drug policies and their impact on youth substance use. Health Promot Int 2004;19:227-34.

18 Department for Education. The importance of teaching: cchools white paper. Stationery Office: London, 2011

19 Bonell C, Harden A, Wells $\mathrm{H}$, et al. Systematic review of the effects of schools and school-environment interventions on health: protocol for evidence mapping and syntheses. BMC Public Health 2011;11:453.

20 Bonell C, Jamal F, Harden A, et al. Systematic review of the effects of schools and school environment interventions on health: evidence mapping and synthesis. Public Health Res 2013;1.

21 Thomas J, Brunton J, Graziosi S. EPPI-Reviewer 4.0: software for research synthesis. EPPI-Centre software. Social Science Research Unit, Institute of Education, London, 2010.

22 Dobbins M, De Corby K, Robeson P, et al. School-based physical activity programs for promoting physical activity and fitness in children and adolescents aged 6-18. Cochrane Database Syst Rev 2009;21:CD007651.

23 Shepherd J, Harden A, Rees R, et al. Young people and healthy eating: a systematic review of barriers and facilitators. Health Educ Res 2006;21:239-57.

24 Shepherd J, Kavanagh J, Picot J, et al. The effectiveness and cost-effectiveness of behavioural interventions for the prevention of sexually transmitted infections in young people aged 13 to 19: a systematic review and economic evaluation. Health Technol Assess Monographs 2010;14:1-206.

25 Valentine JC, Pigott TD, Rothstein HR. How many studies do you need? A primer on statistical power for meta-analysis. J Educ Behav Stat 2010;35:215-47.

26 Flannery DJ, Vazsonyi AT, Liau AK, et al. Initial behavior outcomes for the peacebuilders universal school-based violence prevention program. Dev Psychol 2003;39:292-308.

27 Sallis JF, McKenzie TL, Conway TL, et al. Environmental interventions for eating and physical activity: a randomized controlled trial in middle schools. Am J Prev Med 2003;24:209-17.

28 Dzewaltowski DA, Estabrooks PA, Welk G, et al. Healthy youth places: a randomized controlled trial to determine the effectiveness of facilitating adult and youth leaders to promote physical activity and fruit and vegetable consumption in middle schools. Health Educ Behav 2009;36:583-600.

29 Flay BR, Graumlich S, Segawa E, et al. Effects of 2 prevention programs on high-risk behaviors among African American youth: a randomized trial. Arch Pediatr Adolesc Med 2004;158:377-84.

30 Battistich V. Effects of a school-based program to enhance prosocial development on children's peer relations and social adjustment. J Res Character Educ 2003;1:1-7.

31 Fonagy $\mathrm{P}$, Twemlow SW, Vernberg EM, et al. A cluster randomized controlled trial of child-focused psychiatric consultation and a school systems-focused intervention to reduce aggression. J Child Psychol Psychiatry 2009;50:607-16.

32 Krug EG, Brener ND, Dahlberg $L L$, et al. The impact of an elementary school-based violence prevention program on visits to the school nurse. Am J Prev Med 1997;13:459-63.

33 Ridgers ND, Stratton G, Fairclough SJ, et al. Long-term effects of a playground marking and physical structures on children's recess physical activity levels. Prev Med 2007:44:393-7.

34 Ridgers ND, Stratton GS, Fairclough SJ, et al. Children's physical activity levels during school recess: a quasi-experimental intervention study. Int I Behav Nutr Phys Act 2007:4:1-9.

35 Ridgers ND, Fairclough SJ, Stratton G. Twelve-month effects of a playground intervetion on children's morning and lunchtime recess physical activity levels. J Phys Act Health 2010;7:167-75.

36 Battistich V, Solomon D, Delucchi KD. Effects of a program to enhance prosocial development on adjustment. Annual Meeting of the American Psychological Association; Boston, MA, 1990

37 Battistich V, Solomon D, Watson $M$, et al. Effects of an elementary school program to enhance prosocial behavior on children's cognitive-social problem solving skills and strategies. J Appl Dev Psychol 1989;10:147-69.

38 Bonell $C$, Sorhaindo A, Allen E, et al. Pilot multimethod trial of a school-ethos intervention to reduce substance use: building hypotheses about upstream pathways to prevention. J Adolesc Health 2010;47:555-63.

39 Solomon D, Watson MS, Delucchi KL, et al. Enhancing children's prosocial behavior in the classroom. Am Educ Res J 1988;25:527-54.

40 Stratton G. Promoting children's physical activity in primary school: an intervention study using playground markings. Ergonomics 2000;43:1538-46.

41 Stratton G, Mullan E. The effect of multi-color playground markings on children's physical activity level during recess. Prev Med 2005;41:828-33. 